\title{
Explication of the Methodological Difficulties of Modern Axiology
}

\author{
Victoria V. Kotlyarova \\ Andrey M. Roudenko \\ Marina M. Shubina \\ Yuri A. Shestakov
}

Don State Technical University, Shakhty

Email: rusciense@mail.ru, amrudenko@list.ru

Doi:10.5901/mjss.2015.v6n3s1p477

\begin{abstract}
The authors focus on the group of methodological difficulties of axiology, due to its specific multifaceted subject. One of the major difficulties in the study of values is that there is no unity in the understanding of the essence of value in science. Axiological theories that exist within different philosophical and cultural philosophy of teachings represent a diversity of opinions, conclusions, decisions and methodological approaches. There are several different approaches of consideration of the category of "value", covering different spheres of social life in axiology, in values the elements of the sub-categories and subcategories are distinguished. Methodological pluralism in interpretation and typology of values are analyzing. Theoretical foundations of axiology are formed as a response to two major challenges: existential status of values and their relationship to the facts of reality. The response to these challenges in axiology was pluralization of ways of philosophizing, which is expressed in the generation of a variety of axiologically oriented traditions, based on its own research methodology and leading to the existence of a number of methodological inconsistencies. According to the results of the analysis, the authors concluded that the difficulties of methodological nature, reflecting the diversity of its subject matter, are manifested in the increasing expansion of the research field of axiology, in the unresolved range of the fundamental problems in the axiology in the existing diversity of theories, values and definitions of the concept of "value" itself and etc., this methodological situation requires a rethinking of the methodology and axiology.
\end{abstract}

Keywords: axiology, value, pluralism of axiological theories; typology of values, the methodological principle, methodological difficulties.

\section{Introduction}

Human being is objectively determined, is movable and constituted values, which are laid as a principle - and it does not matter, are they conscious or not - at the heart of any knowledge, especially philosophy.

Nowadays, perhaps, there is not a single humanitarian branch of knowledge, which would not be operated on the notion of value or close to it in meaning such categories as aims, appraisal, value, significance.

Axiology as a philosophical doctrine about the value is one of the main domestic, perhaps, in the foreign world of philosophical discourse, direction of philosophical knowledge. Largely it happens because of the late appearance of axiology in the second half of the nineteenth century, and the historical circumstances that led to the necessity of axiology. The lack of the term "value" in philosophical studies until the second half of the nineteenth century did not mean that philosophical reflection has no idea of value, and, therefore, value-based approach as such. The problem of values in all historical periods was one of the most pressing questions of comprehension of man's inner, spiritual world. The value is a characteristic feature of human life. Since ancient time human being was asked questions that have not lost their significance and are relevant to this day: who am I? Why do I come into the world? What is my place in it? What is the meaning of my life? Being based on what values should I choose my life path, determine the purpose and meaning of my activity, choose the means to achieve them?

The history of the formation of important concepts and ideas should not be abstracted from the premises of their appearance. As a rule, special concepts (and related concepts) appear only when the object of comprehension itself is mainly formed. Appeal to the values becomes an integral part of philosophical doctrines since Antiquity.

In social thought of Ancient Greece the problem was examined by Socrates, Plato, Aristotle. In the Middle Ages a common sense of values, according to most Christian thinkers, was in perfection towards the perfect, divine being. The 
figures of the Renaissance L. Valla, M. Montaigne and others justified the inherent worth of the human person. Social thinkers of the New time R. Descartes, B. Spinoza, John Locke and also the French enlighteners Charles de Montesquieu, K. A. Helvetius were focused on the rational cognition of reality and proved the significance of rationalistic values for a human being (Kagan, M.S., 1997).

Reconstructing the historical and philosophical context of the study of values, it should be noted that an essential prerequisite for the selection of western European axiology as an independent field of scientific knowledge in the second half of XVIII - first half XIX centuries has served the conceptual ideas of the representatives of the German classical philosophy. In 1785 I. Kant wrote "the Foundation for the metaphysics of morals", in which he first considered the concept of "absolute values" (Wert) of net, goodwill, opening up theoretical space for future achievements in axiology.

Further development of ideas about the values is associated with the name of R. Lotze who separates the world of values from the objective world, in opposition with developing in the mid-nineteenth century positivism and biological determinism, which claim pragmatic and physiological rationale spiritual processes (Kotlyarova, V.V., 2014).

The final formation of the western European axiology took place in the first half of the twentieth century and was associated with the emergence of a number of philosophical currents which representatives explored actively the essence of the values First of all, representatives of neokantianism. Baden school of neokantianism (G Rickert, Century, Windelband) laid one of the leading in the nineteenth century directions of axiology - transcendentalism (transcendental objectivism), which is concerned with the nature of values as immutable, eternal, transcendental, empirically real entity, the question of the origin of which remains "taken" and incorrectly staged in general (Rickert, G., 1998). The surge of interest in axiology contributed to working out of different value theories: a phenomenological axiology of M. Scheler, critical ontology of N. Hartmann, axiological normativism of M. Weber, subjective emotivism of $A$. von Meinong, naturalistic psychology of $\mathrm{F}$. Brentano and $\mathrm{V}$. Wundt.

In the first third of the XX century axiology has experienced a real boom, for the studies of value about human, society and the culture allowed to look at them from the inside and impartially, regardless of any socio-economic and party-political preferences (Shokhin, B.K.).

In 60-70 years of the twentieth century, such scholars as K. Bowling, J. Galbraith, A. Maslow, N. Recher, M. Rakic, A. Toffler addressed the issue of values, dedicating their efforts to the formation of applied axiology, which helped to create the basic principles of empirical research. A significant contribution to the development of axiological ideas in the second half of the twentieth century was made by German scientists C. E. Frankl and E. Fromm. The development of modern western European axiology was significantly influenced by the concept of the thinkers of the post-modernists, in particular J.-F. Lyotard, J. Baudrillard.

The Russian humanitarian traditions are characterized by great interest to study of the spiritual world of human. Russian religious philosophy has made a significant contribution to the study of axiological problems (N. A. Berdyaev, F. M. Dostoevsky, I. A. llyin, N. About. Lossky, V. S. Soloviev, P. A. Florensky, Century, Florovsky and others).

In Soviet philosophy axiological issues began were given much attention since the 60-ies of XX century. However, during this period in the works of Travnickova O. G, Zdravomyslova A. G., T. Lyubimova Century, Mamardashvili M. K., Rubinstein, S. L., Stolovich L. N., Tugarinov B. N., Chavchavadze N. C., Chinoy L. A., Adova C. A. and other discussion of the main axiological problems was carried out from the perspective of ethics, aesthetics, social psychology and pedagogy.

However, the authors believe that today researches in the field of values did not lead to the formation of a single position in the understanding of various aspects of this problem.

Axiology passed a difficult way of development, evolving from the philosophy that had significant epistemological consequences - almost all of axiological schools and trends that developed in the framework of philosophy, were based on its own methodology and the research methods, contributing to a "crisis of overproduction", the inevitable result of which was the reduction of the credibility of axiology as a scientific discipline, the existence in it of a number of methodological difficulties, actualizing the questions of their explication.

\section{Methodology of Philosophical Cognition of the Sphere of Values}

\subsection{Literature review and methodology}

The importance of this study is the that philosophical cognition of the sphere of values is based on the developed theoretical and methodological framework, which is based on the writings of thinkers, for which the notion of value has been the subject of a targeted philosophical research subsequently led to the creation of a separate philosophical discipline - axiology. 
This theoretical and methodological heritage in the context of the topic of the thesis has undeniable value, as it is a different kind of attempts to structure, classification and explication of values, including at the level of reflection of the philosophical knowledge of the world of values.

The theoretical-methodological basis of the study is specified by the nature of the object and the subject of research, as well as the challenges that arise from the necessity of philosophical analysis of the concept of "value".

Taking into account the multi-level content of the problem, the research, as axiological in fact, is based on an interdisciplinary and systemic-integrative approach, synthesizing knowledge from different sciences: philosophy, cultural studies, pedagogy, cognitive linguistics. Interdisciplinary vision of the problem allows to identify the communicativefunctional and structural-semiotic aspects of the concept of "value" from the point of view of embodied in it axiological information. Significant group of methods are defined by the methodological principles of structural linguistics, which enable us to develop the content of the problem "language of value", as well as methods of comparative linguistics (verification of research results on the basis of etymological, semantic and phraseological analysis). In the case of solving of complex logico-semantic problems phenomenologi-hermeneutic approach is involved.

The axiological approach is seen as a fundamental condition and principle of explanation and understanding of social existence. It allows to speak about the inherent nature of social life, highlighting methodological installation and rational-discursive intention of thinking about culture as "second nature" where the laws of goal setting and valuesoriented organization person as an individual of his life are predominating.

All researches are based on the fundamental epistemological principle of the unity of the historical and logical. Besides, in the study formal logical methods of abstraction, formalization of system-structural analysis and synthesis, induction and deduction, as well as categories and laws of dialectical logic are used.

The scientific methods of comparison, analogy, analysis, synthesis, and others, as well as general scientific principles of structure, consistency, integrity, history, causation, comprehensiveness and specificity are widely used (Rudenko, A.M., 2012). All mentioned methods as a means of intellectual understanding of the subject of the research allow to identify the nature and the specifics of the problem and to formulate approaches and ways of its solution.

Expose of methodological problems of modern axiology requires integration of efforts of scientists from different fields of knowledge. Characteristically, the interests of many researchers are associated with the logical analysis of concepts of values, as well as with the examining the role of values in the dynamics of society. In this sense a kind of ideals of scientific studies in the philosophy of science, proposed by A. P. Ogurtsova (controversial named "axiological models"), stages of formation of the subject of cognition proposed by A. A. Tikhonov, "the axiological dimension of epistemology of L. A. Mykerinos and others are demonstrative (Kotlyarova, V.V., 2014). Particular interest from the point of view of historical analysis of values cause the works of C. F. Anisimov, L. N. Stolovich and C. K. Alexander Shokhin, who conducted a special analysis of the socio-philosophical teachings of thinkers from different periods. I. I. Dokuchaev defines value as the existential center of human existence and integral generating model of all artifacts of culture, the expression of subjective human place in the cosmos. To this point of view adjoin A. M. works.

As the optimal method for studying of values in the period of classical axiology was proposed and subsequently became widespread method of binary oppositions or dichotomies (objectivism / subjectivism, empiricism / apriorism and others), significantly limiting the consideration of genetic links between the opposed sides. In the western philosophical tradition researchers from different schools tried to find the answer to the question: is the value objective or subjective. It seems that this alternative has no unique solution. This is because the value cannot exist without the object (object) belonging to the outside world, and without a subject (person), because the evaluation of the latter phenomenon is valuable or invaluable.

In non-classical period axiology the representatives of phenomenology (U. Clashes, E. Strecker, P. Jansen, K. the held) and existentialism (K. Jaspers, M. Heidegger, J.-P. Sartre) called for "deobjectivization" of values an approach were manifested, determining the dichotomy as the principle of existential analysis of the philosophical works about the value field. In the positivist tradition, despite the frequent use of "normative" terminology, for a long time prevailed "naturalistic" (when a specific empirically existing "things" have values, objects, facts, events, personality) or subjectively-emotivists interpretation of values and value-relations.

Most modern scientists are united in recognition of the high role of values in processes of socio-cultural genesis. In General, in the history of philosophical and axiological thoughts, on the one hand, a rich tradition research of axiological problems related to the study of values, estimation, value consciousness was formed. However, these studies are conducted on different conceptual basis for the difference in values. Diversity of ideas, evaluations, undoubtedly, should be the norm for science, but it does not negate the problem of creating a synthesis of concepts, which themselves can represent the different positions. 


\subsection{The overall structure of philosophical knowledge of philosophical cognition of the sphere of values}

In philosophy by the beginning of the XXI century, there remains not a single serious theory, which has not designated its relationship to the values, their specificity and role in human life and society, and, therefore, do not form a specific axiological concept.

Any axiological research begins with attempts to determine the concept of the term "value". In a metaphysical approach something existing in itself "Kingdom values" is materializing, transcendental to reality and attaining by a person directly by discretion. At existential and anthropological approach values are derived from the conditions of human existence and from those primary needs, which are defined by these conditions. At sociological approach values are derived from the conditions of existence of the community and the needs of the community in their own reproduction and stable development. This shows the relationship of the methodological problems of modern axiology with the specifics of the category of "value". Scientific publications only confirm the fact of inconsistency of trying to develop modern strategies in the study of values (Rudenko, A.M. and Ivin, A.A., 2010). Having received today dualistic epistemological status the concept of "value" has received a large number of definitions, characteristics and comparisons, which supplement each other, as it was noted earlier in our studies, there are more than twenty definitions of value in the modern Russian reference literature (Kotlyarov, V.C., 2013).

This methodological situation is described by outstanding axiologist of modernity N. Hartmann in his report "the Problem of values in modern philosophy" at the VIII International Congress of philosophy, labeling in different forms "the basic question of axiology": are there values existing regardless of the opinions of people, from their assessments; whether they are absolute or relative; whether it is necessary to associate values with their significance for people, in other words, whether they depends from the state of affairs in the real world (The problem of value in philosophy, 1966).

Till now one of the research stubs in axiology is the situation, when the fundamental concepts of the scientific discipline mutually define each other: talking about "values", "goods", "use", "satisfaction", "interests", "desires", and even "fun". Existing in philosophy not recognition of valueablel, cognitive and utilitarian evaluations is caused by the phenomenon of polysemy, and in the same judgment about any phenomenon existing estimates may be simultaneous as "it's sign" and may contradict and oppose each other. Currently the problem of the"value" concept has developed many philosophical theories that take into account only one of any item. The existence of such a huge number of theories on the problem of values does not bring us closer to the answer, on the contrary, it seems that the question about the essence of value is metaphysical, irrational, never solvable problem.

In the epistemological aspect value is a necessary element of the learning process. In the anthropological aspect values are the result of existential meanings, which fill with the significance human existence. As rightly notes $A$. M. Rudenko "The variety of folk culture, mentality and concrete everyday life of man are reflected In values" (Rudenko, A.M., 2010). In praxeological clue values express the activity of the subject, in this aspect the difference of values from the ideal as a result of an act of volition of active personality is showed most obviously. Value is detected only during activity, thanks to the assessment, which can be both actual and potential. The value may be considered in the hermeneutic aspect, which allows to interpret the processes of existence, defining only one of many possible meanings in accordance with the existing subjective criteria.

The most important methodological problem of modern axiology is the question of typology of values and criteria of the typology. This problem, of course, correlates with the question about the nature of values. The authors believe that the question of the typology of values is not fundamentally solved "fundamental question of axiology", because axiology as an integrative field of philosophy is formed at the junction of a number of different schools and styles, having created its own traditions in the study of values.

Researchers working in line with one of the scientific school are characterized by the fact that they own a particular integrated set of methods, which has a number of very specific certain theoretical concepts. The main problem is the impossibility of a unified classification of values, it can be based on completely different criteria, each of which has not only obvious advantages, but also limitations on its application. It is evident that outside of this scientific school for scientists, as a rule, it is difficult to meet its leading ideas, until associated with it concept would not be perceived.

The most important methodological problem of modern axiology has become not only a question of typology of values and criteria of the typology, but also the problem of typology of axiological theories. This is appropriate to illustrate with examples. And if in the early twentieth century American philosopher and psychologist of German origin G. Munsterberg offers one of the first typologies of values, according to which values are shared on the value of life and cultural values, by the seventies of the twentieth century classification of axiological theories are already proposed.

Classics of axiology emphasize plurality of typologies of axiological theories, for example N. O. Lossky distinguishes three currents: currents, objectivist and theory, deriving from the interaction of subject and object (Lossky, 
N.O., 1931). M. S. Kagan defines in axiology seven streams: psychological, naturalistic, sociological, semantic, objectiveontological, phenomenological, existential and theological (Kagan, M. S. 1997). Recently deceased Professor of University of Tartu, L. N. Stolovich, analyzing the plurality of axiological typologies, emphasizes that actually it is impossible to enumerate them, because in each of the national philosophical tradition there are its own typology (Stolovich, L.N., 1994).

Let's stay on another important characteristic of methodological problems of axiology. Modern axiology experiences a kind of "crisis of overproduction", a natural result of which is the reduction of the authority of axiology as a scientific discipline. In many modern studies the identity of the concepts of value and axiology is recorded, in some studies dual notions are found: "axiological values." Especially "sin" in this dual application of the terms the works done at the intersection of pedagogy and philosophy.

In our opinion, active usage of the term "problem of values" and the term "axiology" in the best case as an example of some common cultural orientation for our time, a kind of axiological "fashion". This existing "fashion" on axiology has not led to a leveling of methodological and theoretical issues, outlining a wide range, starting with the unambiguous definition of "value" and the specific value relationships and ending with the recognition of the situation of axiology in the overall structure of philosophical knowledge.

\section{Results of Explication of the Methodological Difficulties}

Our results of explication of the methodological difficulties of modern axiology complement the study of other contemporary researchers involved in the analysis of this problem. So, the most authoritative Russian philosopher C. K. Shokhin notes that the value is becoming a prestigious widespread and respectable concept in modern philosophical vocabulary and with it, one of the most popular, even populist. Currently, the process of incrementing of new approaches, research directions in axiology continues. C. K. Shokhin justifies the existence of the modern axiology three directions: the axiological naturalism (the value is derived from interest, necessity, needs, motives), phenomenological axiology (value judgments are not empirical but a priori synthetic) and analytic philosophy (actually there is only the act of assessment, which may in various ways be studied by psychology, sociology, metaxiology etc.) (Shokhin, B.K., 2006). Well-known Russian specialist in the field of philosophy of science, history of philosophy and epistemology L. A. Mikeshin suggests splitting of axiological concepts at the abstract - universal or transcendentalists and empirically-dispositional (Mikeshin, L.A., 2009).

We cannot say that in axiology there were no attempted to neutralize the identified difficulties and to form an interdisciplinary theory of values and define the concept of "value", but these studies sooner or later was correlated with only one of any known axiological approach.

The idea of the value as an interdisciplinary concept is putted forward by the author of numerous scientific papers on existential psychology D. A. Leontiev, and suggests to develop a common, unified definition and context of the use of this concept, taken into account its different interpretations. The philosopher applies dimensionally method, borrowed from V. Frankl. His research task D. A. Leontiev determines in building a common space of different definitions letting to fix private projection of such a "complex multidimensional object - which, outside of any doubt, is value" (Leontiev, D.A., 1996).

In the opinion of the authors, the concept of D. A. Leontiev does not solve the methodological problems of developing a universal, interdisciplinary theory of values, taking into account all the diverse manifestations of values. Theoretical constructions of D. A. Leontiev are close on the one hand, to the phenomenological interpretation of values, interpreting the values as a set of different characteristics. From this point of view, values describe the various phenomena of reality, and their number is huge, in contrast to $\mathrm{D}$. A. Leontiev assertion. On the other hand - the position of $D$. A. Leontiev seems to be modification of the psychological approach to the interpretation of the values, because values in his concept perform meaningful and motivational function.

One of the areas of modern axiology, leveling its methodological difficulties, is the idea of interdisciplinary synthesis and application of synergetic methodology in the field of axiology.

M. S. Kagan is one of the first Russian axiologists who attempted application of the synergetic approach to culture and values. Culture in system-synergetic understanding is a complicated, self-organizing system, the trajectory of which cannot be described either in terms of linear thinking, nor in terms of the relativistic consciousness.

In an attempt to understand the relationship between complex and simple as the particular media unchanging and invariant traits for complex, it is necessary to reduce the complex to the simple, highest to lowest. Thus, M. S. Kagan believes that this way of learning helps to get knowledge of the foundations of this structure, but not the knowledge itself (Kagan, M.C., 1996). M. S. Kagan notes the need to supplement the system-synergetic approach in order to provide a 
holistic, integrated vision of cultural-historical process. Based on achieved by theoretical thought in the twentieth century level of knowledge of the existence, M. S. Kagan findes modern simple systems thinking, and synergistic understanding of development processes in nature, society, culture, human life, and philosophical reflection as a way of understanding the methodological principles of self-organization of cognitive activity. It should be noted that M. S. Kagan pointed out to the existence of a number of problems associated with the use of a synergistic approach to research values. Recognizing the necessity of interpenetration of methodological approaches of natural sciences and humanities, the scientist points to the danger of mechanical transfer of synergetic methodology in the sphere of humanitarian knowledge.

At the same time, these ideas do not contradict, but systematically and organically complement the survey results, which were obtained.

\section{Conclusion}

Thus, in the study the authors concluded:

- in the process of disciplinary differentiation of axiology as part of socio-humanitarian knowledge, arose methodological in nature difficulties, reflecting the diversity of its subject;

- the methodological difficulties are manifested in the increasing expansion of the research field of axiology, in the unresolved range of the fundamental problems delivered in the axiology in the existing diversity of theories, values and definitions of the concept of "value" and so on;

- the presence of a fixed methodological difficulties in axiology turns the questions of systematization of theoretical constructs in the most important problem requiring reconsideration of its classical and non-classical methodology, the crisis which motivates us to search her post-non-classical forms.

\section{References}

Kagan, M. S. 1997. Philosophical theory of value. - SPb.: LLP TC "Petropolis"

Kotlyarova, V.V., 2014. Transcendental paradigm classic axiology //Economic and Humanities research regions. No. 4.

Rickert,G, 1998. Tow paths of Knowledge. - SPb. (New ideas in philosophy. Sat. 7 : Theory of knowledge III.)

Shokhin, B.K., 1998. Classical philosophy values: background, problems, results // alpha and omega. № 3 (№ 17).

Rudenko, A.M., 2012. Socialisterna concept semantically intentionality of human existence: Dis...Doc. filos. Sciences: 09.00.13 - Rostovon-don.

Kotlyarova, V.V., 2014. Dichotomous approach to non-classical axiology //Economic and Humanities research regions. No. 6.

Rudenko, A.M., 2012. Socialitelife values semantically intentionality of human existence // Economic and Humanities research regions. No.3.

Ivin, A.A., 2010. Modern axiology: some emerging issues // journal of Philosophy. №. 1.

Kotlyarov, V.C., 2013. Methodological problems of modern axiology // Humanitarian and socio-economic science. No. 1.

The problem of value in philosophy, 1966 / edited by A. G. Kharchev (editor), T. N. Hornstein, M. A. Kisses and B. N. Tugarinov. - M.-L., $266 \mathrm{pp}$.

Rudenko, A.M., 2010. Implementation problems of life meaning of intentionality of Asistencia person in the modern socio-cultural reality // Humanities and social Sciences. . No. 3.

Lossky, N.O., 1931. The value and existence. - Paris: YMCA-PRESS.

Stolovich, L.N., 1994. Beauty. Good. The truth: an essay on the history of aesthetic axiology. - M.: The Republic.

Shokhin, B.K., 2006. Philosophy of values and early axiological thought. - M., Publ PFUR.

Mikeshin, L.A., 2009. Modern development of the concept of "value" // Values and meanings. No. 1.

Leontiev, D.A., 1996. Value as an interdisciplinary concept: experience multidimensional reconstruction of // problems of philosophy. No. 4

Kagan, M.C., 1996. Philosophy of culture. - S-P.: LLP TC "Petropolis". 